\title{
Selezione dalla mailing-list SociSin del Supplemento "Ha ancora senso fare emodialisi domiciliare nel 2001?"
}

\author{
www.sin-italia.org/mlist
}

\section{Claudio Ronco, Vicenza}

Cari Amici, la domanda reale non è se "ha ancora senso inviare a domicilio per la dialisi un paziente" ma secondo me sarebbe invece: "è ancora possibile inviare a domicilio dei pazienti in emodialisi?" Intanto va chiarito che la dialisi domiciliare deve includere a tutti gli effetti la peritoneale e quindi quello che abbiamo perduto di trattamenti extracorporei domiciliari lo abbiamo recuperato in trattamenti peritoneali. Secondo, il problema riguarda l'organizzazione dei nuclei familiari che risulta sempre più disgregata e quindi non vi sono motivazioni o peggio assunzioni di responsabilità per mantenere a casa un paziente. Come sempre succede due importanti aspetti guideranno le decisioni a venire e sono l'impatto economico e la tecnologia applicata. Credo comunque che non sia possibile pensare a un programma con pochi malati a domicilio per semplici questioni organizzative: o esiste un programa ampio e codificato oppure il costo di qualche trattamento sporadico diventa eccessivo in termini di risorse e di personale umano. Va comunque segnalato che la penetrazione della peritoneale è ancora scarsa e non si capisce come un programma di dialisi domiciliare possa esistere senza avere una opzione per la peritoneale. La domanda finale sarebbe poi: "quale trattamento vorresti per te stesso?" In questo caso risponderei, per quanto mi riguarda, che vorrei iniziare con una peritoneale a casa e passare successivamente a una giornaliera serale a casa (se avessi qualcuno che mi supporta) se il peritoneo diventasse inagibile. Il professor Stiller di Aachen aveva scelto questo protocollo ed è in dialisi da più di 30 anni con una qualità di vita eccellente e un basso numero di complicazioni. Sarebbe questo applicabile a tutti i pazienti? No certo, ma il concetto di poter personalizzare il trattamento includendo una simile "schedule" è sicuramente attraente e dovrebbe essere esplorato con atteggiamento possibilistico.

\section{Amedeo De Vecchi, Milano}

Caro Claudio e cari colleghi, credo che il problema della dialisi sia ben riassunto dalla tua lettera: noi per il paziente uremico abbiamo diverse armi con divera efficacia, diverso "peso" e diverso costo per la qualità di vita. Dovremmo sempre cercare di utilizzare i trattamenti in modo da ottenere la massima qualità di vita, purché non vi siano controindicazioni: per prima la dialisi peritoneale, possibilmente incrementale, poi l'emodialisi domiciliare serale quotidiana, poi l'emodialisi in CAL poi in Centro. In tutto questo dovrebbero inserirsi al più presto uno o più trapianti. Il passaggio da una all'altra dialisi dovrebbe essere estremamente favorito in caso di necessità cliniche o sociali, queste ultime anche minime. È inutile che quasi tutti i nefrologi continuino a pensare a una dialisi, sarebbe come se un cavaliere medievale in un torneo, rotta la lancia, si arrendesse senza pensare di adoperare il pugnale, la spada, la mazza ecc. Credo che finché non supereremo questo gap culturale difficilmente otterremo il miglior risultato possibile nei nostri pazienti e spesso spenderemo inutilmente di più. Credo che tutti dobbiamo farci sempre mille esami di coscienza cercando di metterci nei panni del paziente. Buon anno a tutti, Amedeo De Vecchi.

\section{LAMBerto Oldrizzi, Verona}

Desidero replicare a De Vecchi. Vorrei ricordare che le realtà sanitarie, la disponibilità di mezzi, economica e, soprattutto, di personale (infermieristico e medico) sono profondamente diverse non solo da Regione a Regione, ma anche nei piccoli Centri rispetto alle grandi città. Non si tratta quindi solo di esami di coscienza, gap culturali e tornei medievali, ma, spesso e volentieri, di semplici possibilità organizzative.

Cerchiamo di stare con i piedi per terra! 